\title{
Statin-induced inhibition of the Rho-signaling pathway activates PPAR $\alpha$ and induces HDL apoA-I
}

\author{
Geneviève Martin, Hélène Duez, Christophe Blanquart, Vincent Berezowski, \\ Philippe Poulain, Jean-Charles Fruchart, Jamila Najib-Fruchart, Corine Glineur, \\ and Bart Staels
}

Département d'Athérosclérose, UR 545 Institut National de la Santé et de la Recherche Médicale (INSERM),
Institut Pasteur de Lille and Faculté de Pharmacie, Universite de Lille II, Lille, France

Address correspondence to: Bart Staels, UR 545 INSERM, Institut Pasteur de Lille, 1 Rue du Prof. Calmette B.P.245, 59019 Lille Cédex, France. Phone: 33-3-20-87-73-88; Fax: 33-3-20-87-71-98; E-mail: Bart.Staels@pasteur-lille.fr.

Received for publication July 25, 2000, and accepted in revised form April 23, 2001.

\begin{abstract}
Statins are inhibitors of the rate-limiting enzyme in cholesterol synthesis, 3-hydroxy-3-methylglutaryl coenzyme A (HMG-CoA) reductase. In addition to reducing LDL cholesterol, statin treatment increases the levels of the antiatherogenic HDL and its major apolipoprotein apoA-I. Here, we investigated the molecular mechanisms of apoA-I regulation by statins. Treatment with statins increased apoA-I mRNA levels in human HepG2 hepatoma cells, and this effect was reversed by the addition of mevalonate, implicating HMG-CoA reductase as the relevant target of these drugs. Pretreatment with Actinomycin D abolished the increase of apoA-I mRNA, indicating that statins act at the transcriptional level. Indeed, statins increased the human apoA-I promoter activity in transfected cells, and we have identified a statin response element that coincides with a PPAR $\alpha$ response element known to confer fibrate responsiveness to this gene. The statin effect could be abolished not only by mevalonate, but also by geranylgeranyl pyrophosphate, whereas inhibition of geranylgeranyl transferase activity or treatment with an inhibitor of the Rho GTP-binding protein family increased PPAR $\alpha$ activity. Using dominant negative forms of these proteins, we found that Rho A itself mediates this response. Because cotreatment with statins and fibrates activated PPAR $\alpha$ in a synergistic manner, these observations provide a molecular basis for combination treatment with statins and fibrates in coronary heart disease.
\end{abstract}

J. Clin. Invest. 107:1423-1432 (2001).

\section{Introduction}

Clinical benefits of cholesterol reduction have been established in large-scale primary and secondary intervention trials with statins, demonstrating that treatment with these drugs results in decreased morbidity and mortality related to coronary heart disease (CHD) $(1,2)$. Statins are competitive inhibitors of 3-hydroxy3-methylglutaryl coenzyme A (HMG-CoA) reductase, the rate-limiting step in cholesterol synthesis, thus decreasing endogenous cholesterol synthesis. The lowering of intracellular cholesterol levels leads to the activation of sterol regulatory element-binding protein (SREBP) transcription factors, which activate the transcription of a number of genes involved in the clearance of LDL particles from plasma, such as the LDL receptor (3). Despite a significant reduction of LDL cholesterol, a number of patients still have clinical events likely due to the contribution of other factors to the atherogenic process (4). The new generation of more potent statins, such as atorvastatin, cerivastatin, and pitavastatin (NK-104), also lower plasma triglycerides due to a diminished VLDL production, probably as a consequence of less availability of cholesterol for VLDL assembly (5). In addition, they may lower triglycerides due to a strong induction of receptor-mediated remnant clearance (5). Furthermore, statin treatment also increases plasma concentrations of antiatherogenic HDL cholesterol and apoA-I (6), which play a crucial role in reverse-cholesterol transport (7). These observations are likely of clinical relevance, since high levels of HDL and apoA-I are considered to protect against development of CHD (8-10). Moreover, studies in transgenic animals have revealed that overexpression of human apoA-I in mice (11) and rabbits (12) increases plasma apoA-I and HDL cholesterol levels, leading to the inhibition of atherogenesis in dietary and genetically induced animal models of atherosclerosis.

In addition to their potent action on plasma lipid concentrations, statins exert pleiotropic properties and interfere with different vascular events leading to the formation of atherosclerotic lesions, effects that may contribute to their beneficial effects on CHD $(13,14)$. Statins may exert direct antiatherosclerotic effects by acting on smooth muscle cells (SMCs), endothelial cells, and macrophages $(15,16)$. Statins prevent SMC migration and proliferation (15) and suppress lipopolysaccharide-induced ICAM-1 expression in bovine aortic endothelial cells (17). Statins may also increase fibrinolytic capacity by inhibiting the expression of PAI-1 in SMCs and endothelial cells while increasing expression of tPA in endothelial cells (18) and suppressing tissue factor expression in cultured 
human macrophages (19). Therefore, statins may interfere with the formation and progression of the atherosclerotic plaque as well as with thrombotic events in hyperlipidemic patients, independently of their ability to reduce plasma cholesterol (20). Beneficial effects of statins against atherosclerosis could be attributed to their ability to suppress the synthesis of mevalonate (16) or downstream products of mevalonate such as isoprenoid intermediates. In vitro experiments demonstrated that the effects of statins on vascular cells were prevented by the addition of mevalonate, and isoprenoids such as farnesyl pyrophosphate (Fpp) or geranylgeranyl pyrophosphate (GGpp) have been implicated in this action $(17,21)$. In endothelial cells, the effects of statins were mimicked by C3 exoenzyme, an inhibitor of Rho activity (21). As such statins inhibit the prenylation of proteins, such as Ras and Rho that activate the mitogen-activated protein (MAP) kinase cascade or NF- $\kappa$ B pathway, leading to anti-inflammatory, antiproliferative and antithrombotic effects.

PPARs belong to the superfamily of nuclear receptors that are ligand-activated transcription factors (22). Fatty acid derivatives and eicosanoids were identified as natural ligands for PPARs $(23,24)$. Following heterodimerization with the 9-cis retinoic acid receptor RXR, PPARs bind to specific PPAR response elements (PPREs) in the regulatory regions of target genes (25). Among the three different PPAR subtypes identified thus far, PPAR $\alpha$, PPAR $\beta$ (NUC- 1 or PPAR $\delta$ ), and PPAR $\gamma, \operatorname{PPAR} \alpha$ mediates the lipid-lowering activity of the fibrate drugs (26). PPAR $\alpha$ is considered a major regulator of intra- and extracellular fatty acid metabolism. In humans, fibrate activation of PPAR $\alpha$ increases plasma levels of HDL, decreases VLDL synthesis and secretion, and reduces triglyceride levels. Furthermore, PPAR $\alpha$ mediates the anti-inflammatory actions of fibrates, such as fenofibrate, at the level of the vascular wall $(27,28)$. PPAR $\alpha$ has been shown to exert these effects by negatively interfering with NF-KB and activator protein-1 (AP-1) (25).

Since statins increase HDL and apoA-I plasma levels, the first goal of this study was to determine the molecular mechanism leading to increased apoA-I production under HMG-CoA reductase inhibition. Furthermore, because of the striking parallel between the effects of fibrate PPAR $\alpha$ agonists and statins on lipoprotein metabolism and inflammation, we hypothesized the existence of a common mechanism via cross-talk of their pathways. Our results show that statins increase human apoA-I mRNA levels through activation of its promoter. Interestingly, statins activate PPAR $\alpha$ by inhibiting the Rho A signal transduction pathway. These results provide a molecular basis for the increase in HDL levels in response to statins and establish a positive cross-talk between the statin and fibrate pathways.

\section{Methods}

Materials. Cerivastatin was kindly provided by Bayer AG (Wuppertal, Germany). Pitavastatin was provided by Nissan Chemical Industries Ltd. (Tokyo, Japan).
Fenofibric acid was kindly provided by A. Edgar (Fournier, Dijon, France). Actinomycin D was from Roche Diagnostics (Meylan, France). Fpp and GGpp were purchased from Biomol Research Laboratories (Plymouth Meeting, Pennsylvania, USA). Mevalonate, nordihydroguaiaretic acid (NDGA), farnesol, squalene, 22(R)- and 22(S)-hydroxycholesterol, cerulenin, and chenodeoxycholic acid (CDCA) were purchased from Sigma-Aldrich (St. Quentin, France). Farnesyl transferase inhibitor and geranylgeranyl transferase inhibitor were from CN Biosciences Inc. (La Jolla, California, USA). Ketoprofen was from Aventis (Vitry, France). Arachidonyltrifluoromethyl ketone (AACOCF3) was from Euromedex (Souffelweyersheim, France). Etomoxir was a kind gift from R. Berge (University of Bergen, Bergen, Norway).

Cell culture and RNA analysis. Human hepatoma HepG2 cells (American Type Culture Collection, Rockville, Maryland, USA) were maintained in DMEM supplemented with $10 \% \mathrm{FCS}$ at $37^{\circ} \mathrm{C}$ in a humidified atmosphere of $5 \% \mathrm{CO}_{2}$. Medium was changed every 2 days. At $80 \%$ confluence, the medium was replaced by a serumfree medium for 18 hours before treatment with statins to avoid interference of serum components. Cerivastatin was dissolved in water and pitavastatin in DMSO. Cells were subsequently incubated with the indicated compounds for the indicated period of time. At the end of the treatment period, cells were washed with ice-cold PBS and homogenized in $4 \mathrm{M}$ guanidinium isothiocyanate. RNA preparation, Northern blot hybridizations, and quantifications were performed as described previously (29). Human apoA-I (30) and 36B4 cDNAs were used as probes.

Transient transfection assays. All the transfection experiments were performed in triplicate in human hepatoma HepG2 or in rabbit kidney RK13 cells. Cells were transfected in serum-free medium using the lipofectant method with a mixture of plasmids that contained, in addition to the firefly luciferase reporter and expression vectors, a Renilla luciferase expression vector as a control for transfection efficiency. All samples were complemented with an equal total amount of DNA using pBluescript empty vector. After 2 hours, cells were incubated for another 40 hours with the indicated stimuli dissolved in their respective solvents and added at the indicated concentrations in medium supplemented with Ultroser serum, which is devoid of sterols and lipids (Biosepra SA, Cergy-Pontoise, France). The human apoA-I promoter $(-2051,+91)$ and deletion ($256,+91)$ and $(-128,+91)$, as well as wild-type $A\left(A_{w t}\right)$ and mutant $\mathrm{A}\left(\mathrm{A}_{\mathrm{mt}}\right)$ sites in front of the minimal apoA-I promoter $\left(\mathrm{P}_{\min }\right)$ constructs were described previously (31). The A-site mutated apoA-I promoter construct $(-256,+91)$ was obtained using the QuickChange sitedirected mutagenesis kit (Stratagene, La Jolla, California, USA) with the primer $A_{\text {mt }}\left(5^{\prime}\right.$-CTC CCG CCC CCA CTG ATC CCT TGT CCC CTG CCC TGC AGC CCC CG-3'). The consensus DR1 PPRE containing reporter plasmids and the human PPAR $\alpha$ expression vectors were 
described previously $(27,32)$. The plasmids pGal5TKpGL3, Gal4-hPPAR $\alpha$ LBD, and pGal4- $\varnothing$ have been described previously (33). The dominant negative constructs of Rho A, Cdc42, and Rac were kindly provided by P. Fort (Centre National de la Recherche Scientifique [CNRS], Unité Mixte de Recherche [UMR] 5535, Montpellier, France) and P. Chavrier (Institut Curie, CNRSUMR144, Paris, France) (34).

Kinase assay. Purified PPAR $\alpha$ protein was produced using the IMPACT-T7 system (New England Biolabs, Beverly, Massachusetts, USA). HepG2 cells were cultured in the presence of mevalonate $(3 \mathrm{mM})$ or cerivastatin $(5 \mu \mathrm{M})$ for 48 hours. Cells were lysed in kinase buffer (25 mM HEPES, pH 7.5, $100 \mathrm{mM} \mathrm{NaCl}, 1.5 \mathrm{mM}$ $\mathrm{MgCl}_{2}, 0.5 \mathrm{mM}$ EGTA, $0.25 \mathrm{mM}$ EDTA, $0.1 \%$ Nonidet P-40 [NP40], and $10 \mathrm{mM} \mathrm{NaF})$. Purified PPAR $\alpha(2 \mu \mathrm{g})$ protein was incubated with cell extracts $(5 \mu \mathrm{g})$ in the presence of $\gamma\left[{ }^{32} \mathrm{P}\right] \mathrm{ATP}(5 \mu \mathrm{Ci})$ for 30 minutes at $30^{\circ} \mathrm{C}$ in reaction buffer $(50 \mathrm{mM}$ HEPES, $\mathrm{pH} 7.5,100 \mathrm{mM} \mathrm{KCl}$, $10 \mathrm{mM} \mathrm{MgCl} 2,1 \mathrm{mM} \mathrm{MnCl}$, $20 \mu \mathrm{M}$ ATP, $500 \mu \mathrm{M}$ EGTA, $25 \mathrm{mM} \beta$-glycerophosphate, and $1 \mathrm{mM}$ ortho- vanadate). PPAR $\alpha$ protein was immunoprecipitated using a polyclonal $\mathrm{Ab}$ raised against a peptide containing amino acids 10-56 of human PPAR $\alpha$ (35) and consecutively washed with $1 \mathrm{ml}$ RIPA $(100 \mathrm{mM}$ Tris- $\mathrm{HCl}$, $150 \mathrm{mM} \mathrm{NaCl}, 2 \mathrm{mM}$ EDTA, $0.5 \%$ NP40, 0.5\% deoxycholate, and $0.1 \%$ SDS), $1 \mathrm{ml}$ RIPA-NaCl $1 \mathrm{M}, 1 \mathrm{ml}$ RIPA-TNE (10 mM Tris- $\mathrm{HCl}, \mathrm{pH}$ 7.4, $150 \mathrm{mM} \mathrm{NaCl}, 2$ $\mathrm{mM}$ EDTA), and $1 \mathrm{ml}$ TNE. Then samples were boiled in Laemmli buffer and loaded on 10\% SDS-PAGE. After the run, the gel was treated for 30 minutes in neutral fixer ( $40 \%$ methanol, $3.5 \%$ paraformaldehyde), washed with $10 \%$ ethanol, $4 \%$ glycerol, and dried. The gel was stained with Ponceau S solution as control, and phosphorylated PPAR $\alpha$ was visualized by autoradiography.

Statistical analysis. ANOVA, to evaluate global significance, followed by Scheffé test, to analyze for significant differences between groups, were performed.

\section{Results}

Statins induce buman apoA-I mRNA in human bepatoma HepG2 cells. Since statin treatment results in increased a

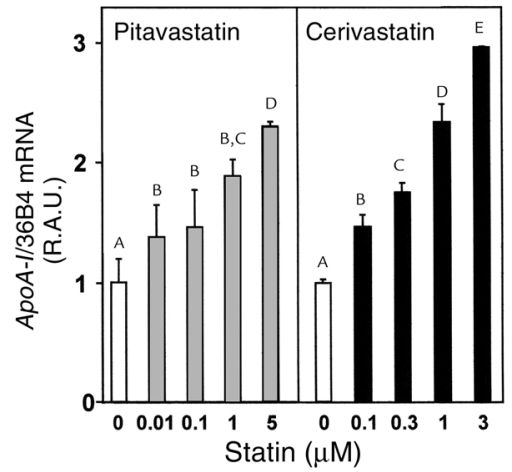

C

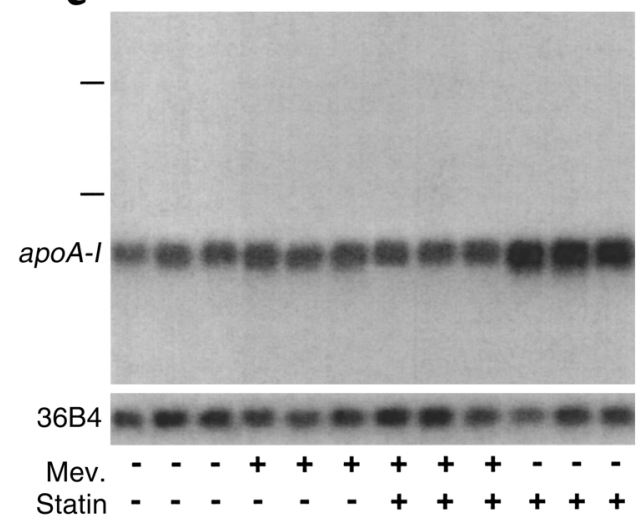

b
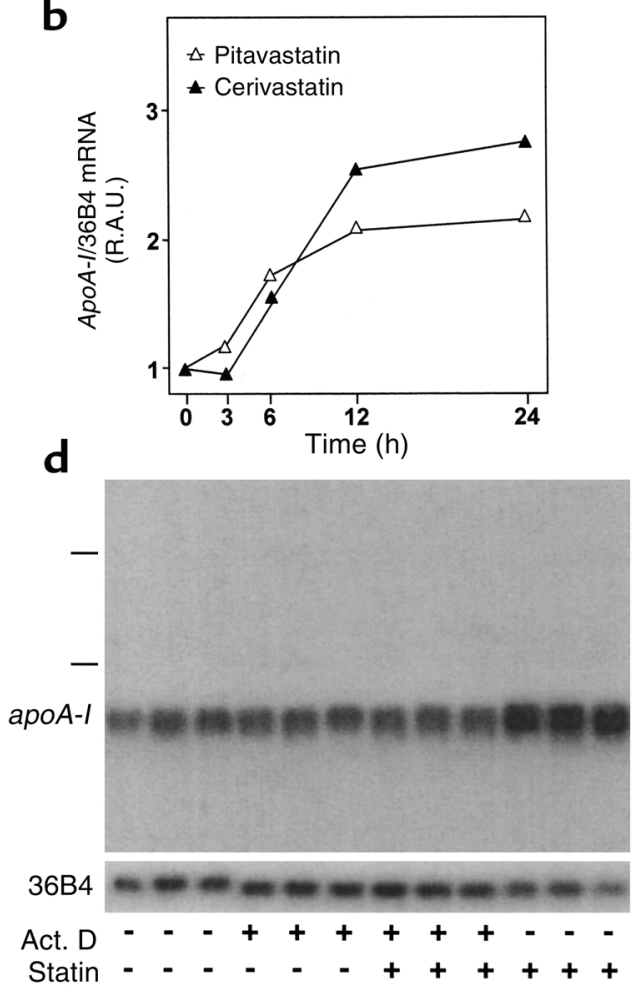

Figure 1

Statins induce apoA-I gene expression at the transcriptional level in HepG2 cells by inhibition of HMG-CoA reductase. (a) Statins induce apoAI mRNA in HepG2 cells in a dose-dependent manner. HepG2 cells were treated for 24 hours with the indicated doses of cerivastatin or pitavastatin or appropriate solvent in serum-free medium. RNA levels were quantified as described in Methods and expressed (means \pm SD, $n=3$ /point) relative to the untreated control set at 1 . Values without a common superscript are significantly different, $P<0.05$. R.A.U., relative arbitrary units. (b) Statins induce apoA-I mRNA in HepG2 cells in a time-dependent manner. HepG2 cells were treated for 3, 6, 12, or 24 hours with $5 \mu \mathrm{M}$ of cerivastatin, or pitavastatin, or appropriate solvent in serum-free medium. RNA levels were quantified as described in Methods and expressed relative to the untreated controls at each time point set at 1. (c) Mevalonate reverses the induction of apoA-I mRNA by statins. HepG2 cells were treated for 24 hours with cerivastatin $(5 \mu \mathrm{M})$ and/or mevalonate (Mev.; $3 \mathrm{mM}$ ) or appropriate solvent in serumfree medium. (d) Statins regulate apoA-I gene expression in HepG2 cells at the transcriptional level. HepG2 cells were treated for 24 hours with cerivastatin $(5 \mu \mathrm{M})$ or appropriate solvent in serum-free medium. Actinomycin D (Act. D; $5 \mu \mathrm{g} / \mathrm{ml}$ ) was added to the medium 90 minutes before treatment. For all, total RNA $(10 \mu \mathrm{g})$ was subjected to Northern blot analysis using human apoA-I and 36B4 cDNA probes. 

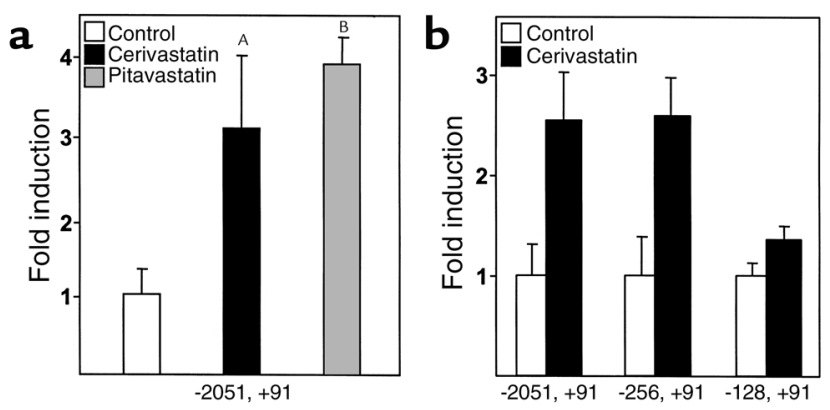

Figure 2

Statins induce human apoA-I promoter activity in HepG2 cells. (a) The human apoA-I promoter $(-2093,+91)$ containing firefly luciferase reporter vector ( $50 \mathrm{ng}$ ) was transfected in HepG2 cells in the presence of a Renilla luciferase reporter vector as internal control (2 ng). After 2 hours of transfection, cells were re-fed with DMEM supplemented with $2 \%$ Ultroser SF in the presence of cerivastatin $(5 \mu \mathrm{M})$, pitavastatin $(5 \mu \mathrm{M})$, or vehicle for 40 hours. Firefly luciferase activities were normalized to Renilla control activities. Values (means \pm SD, $n=3$ ) are expressed relative to controls. Statistically significantly differences between statin-treated and control groups are indicated (Scheffé: ${ }^{A} P<0.05$; $\left.{ }^{B} P<0.01\right)$. (b) The indicated human $a p o A-I$ promoter 5 -deletion constructs ( $50 \mathrm{ng}$ ) were transfected together with the Renilla luciferase expression vector as internal control (2 ng) in HepG2 cells. HepG2 cells were subsequently treated with cerivastatin $(5 \mu \mathrm{M})$ in DMEM supplemented with $2 \%$ Ultroser SF for 40 hours. Firefly luciferase activities were normalized to Renilla control activities. Values (means $\pm \mathrm{SD}, n=3$ ) are expressed relative to controls.

plasma concentrations of apoA-I $(6,36)$, it was investigated whether statins regulate apoA-I at the level of gene expression. Therefore, human HepG2 hepatoma cells were treated with two different statins, and apoAI mRNA levels were analyzed. Treatment with both cerivastatin and pitavastatin increased apoA-I mRNA in a dose-dependent manner, with significant increases already being observed at doses between 0.1 and $1 \mu \mathrm{M}$ (Figure 1a). Furthermore, both statins induced apoA-I mRNA levels in a time-dependent manner. A significant increase was already observed at 6 hours, whereas a maximum was attained at 12 hours (Figure $1 \mathrm{~b}$ ).

To demonstrate that this induction of apoA-I is due to the pharmacological activity of statins on HMG-CoA reductase activity, it was determined whether addition of mevalonate, the product of HMG-CoA reductase, could reverse the induction of apoA-I mRNA by statins. Simultaneous incubation of cerivastatin with mevalonate completely reversed the effect of statins on apoA-I mRNA (Figure 1c). These results indicate that statins exert their effects on apoA-I gene expression by inhibition of HMG-CoA reductase and that downstream products of the mevalonate pathway must be responsible for the observed effects.
To study whether the statin induction of apoA-I mRNA levels occurs at the transcriptional level, the influence of the RNA polymerase II inhibitor actinomycin D on statin-induced apoA-I mRNA was studied in HepG2 cells. The addition of actinomycin D 90 minutes before statin treatment completely abolished the increase in apoA-I mRNA levels by cerivastatin (Figure 1d). These effects of statins on apoA-I gene expression were specific since 36B4 mRNA levels did not change. Thus, statins induce apoA-I mRNA levels by increasing apoA-I gene transcription.

Statins induce apoA-I promoter activity. Since statins induce apoA-I expression at the transcriptional level, it was next studied whether these drugs act by inducing apoA-I promoter activity. Transient transfection experiments in HepG2 cells carried out with a reporter driven by a 2 -kb region of the human apoA-I promoter demonstrated that cerivastatin and pitavastatin significantly (3-fold and 3.5-fold) increased human apoA-I promoter activity (Figure 2a). Next, transfection experiments were performed testing the influence of statin treatment on different $5^{\prime}$-deletion constructs in order to map the statin-response element. The -256-bp proximal apoA-I promoter was induced to an extent similar to that of the $2-\mathrm{kb}$ apoA-I promoter. However, further deletion to position -128 completely abolished the statin effect (Figure $2 \mathrm{~b})$. These results indicate that sequences within the -128-bp to -256-bp promoter region are mediating the statin induction of apoA-I promoter activity.
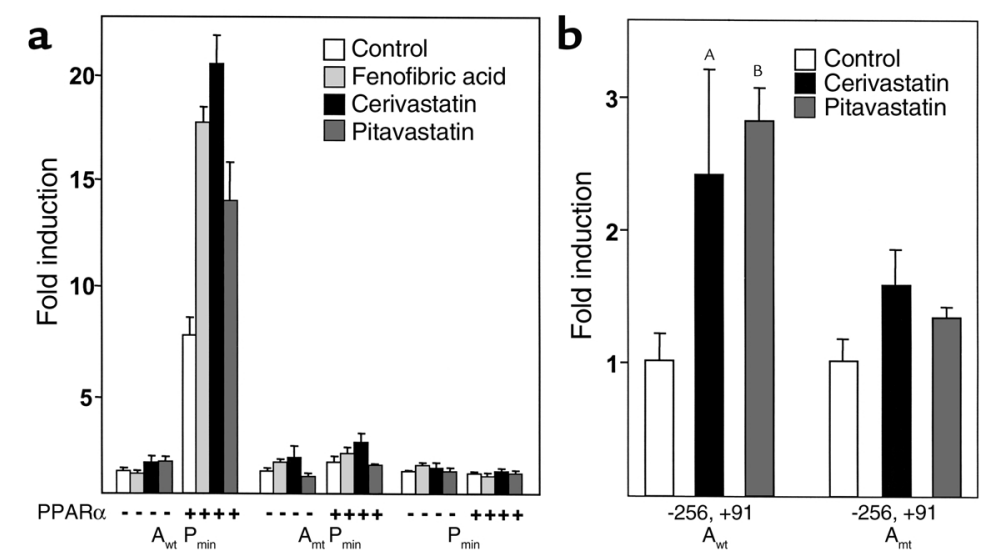

Figure 3

Statins induce human apoA-I A-site activity by PPAR $\alpha$. (a) Reporter plasmids containing the human wild-type and mutated apoA-I A sites cloned upstream of the minimal apoA-I promoter $\left(\mathrm{P}_{\text {min }}\right)(50 \mathrm{ng})$ were transfected in RK13 cells in the presence of cotransfected human PPAR $\alpha$ or empty PSG 5 vector plasmids (30 $\mathrm{ng}$ ) with a Renilla luciferase reporter vector as internal control (2 $\mathrm{ng})$. Cells were treated with cerivastatin $(5 \mu \mathrm{M})$, pitavastatin $(5 \mu \mathrm{M})$, fenofibric acid $(100 \mu \mathrm{M})$, or solvent. Firefly luciferase activities were normalized to Renilla control activities. Values (means $\pm S D, n=3$ ) are expressed relative to controls. (b) Reporter plasmids containing the wild-type $\left(-256,+91 \mathrm{~A}_{\mathrm{wt}}\right)$ or A-site mutated $(-256,+91$ $A_{m t}$ ) apoA-I promoter construct in HepG2 cells with a Renilla luciferase reporter vector as internal control ( $2 \mathrm{ng})$. Cells were treated with cerivastatin $(5 \mu \mathrm{M})$, pitavastatin $(5 \mu \mathrm{M})$, or solvent. Firefly luciferase activities were normalized to Renilla control activities. Values (means $\pm \mathrm{SD}, n=3$ ) are expressed relative to controls. Statistically significantly differences between statin-treated and control groups are indicated (Scheffé: ${ }^{A} P<0.05 ;{ }^{B} P<0.01$ ). 


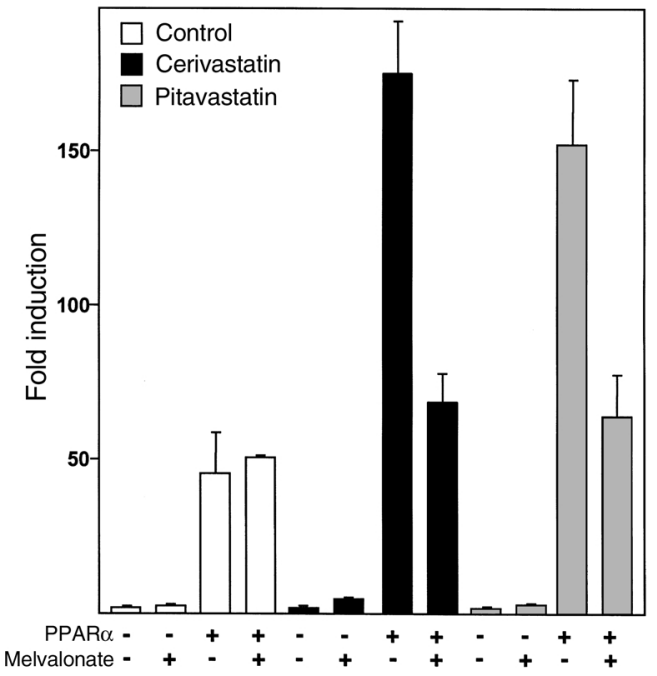

Figure 4

Inhibition of HMG-CoA reductase activity by statins enhances PPAR $\alpha$ activity on a DR- 1 site. RK13 cells were transfected with a reporter construct containing six copies of the direct repeat 1 (DR1) (DR1-TK-Luc) (10 ng) in the presence of cotransfected empty pSG5 or PSG5hPPAR $\alpha$ expression vector (30 $\mathrm{ng}$ ) and a Renilla luciferase reporter vector as internal control ( $2 \mathrm{ng})$. RK13 cells were incubated for an additional 40 hours with cerivastatin $(5 \mu \mathrm{M})$, pitavastatin $(5 \mu \mathrm{M})$, and/or mevalonate $(3 \mathrm{mM})$ in appropriate solvent in DMEM supplemented with $2 \%$ Ultroser SF. Firefly luciferase activities were normalized to Renilla control activities. Values (means $\pm \mathrm{SD}, n=3$ ) are expressed relative to controls.

Statins induce buman apoA-I promoter A-site activity by activating PPAR $\alpha$. Previous studies have demonstrated that fibrates induce the expression of human apoA-I gene by PPAR $\alpha$, which interacts with a positive PPRE located in the A site (31). Since the statin-responsive element of the apoA-I gene is located in the same region, it was next determined whether statins may also act via the PPAR response element identified previously. Therefore, the influence of statins was analyzed on a reporter construct containing the apoA-I PPRE $(-215$, $-195)$ cloned in front of the minimal apoA-I promoter $\left(\mathrm{A}_{w t} \mathrm{P}_{\min }\right)$ in RK13 cells, which are devoid of PPAR $\alpha$ (33). Cerivastatin and pitavastatin did not affect the activity of this construct in the absence of cotransfected PPAR $\alpha$ (Figure 3a). Cotransfection of PPAR $\alpha$ significantly induced the activity of $\mathrm{A}_{w t} \mathrm{P}_{\min }$, and statin treatment resulted in a further enhancement. As a control, the PPAR $\alpha$ ligand fenofibric acid induced apoA-I A-site activity only in the presence of cotransfected PPAR $\alpha$ (Figure 3a). By contrast, a construct containing the A site mutated in its DR2 PPRE sequence $\left(\mathrm{A}_{\mathrm{mt}} \mathrm{P}_{\mathrm{min}}\right)$ was no longer activated by $\operatorname{PPAR} \alpha$, nor by statin, nor fenofibric acid treatment. Similarly, neither PPAR $\alpha$ nor statin treatment enhanced the activity of the minimal apoA-I promoter. These data indicate that the A site, which contains a positive PPAR-response element, is a target for statin induction in the presence of PPAR $\alpha$.

To determine whether statins activate human apoA-I promoter activity via the A site PPRE, the influence of statin treatment on the activity of wild-type and A-site mutated apoA-I promoter was tested next in HepG2 cells (Figure 3b). Again, statin treatment increased wild-type apoA-I promoter activity, but this effect was nearly abolished in the promoter mutated in the A site (Figure $3 \mathrm{~b}$ ).

Statins induce the activity of a consensus PPRE in the presence of PPAR $\alpha$. Since the human apoA-I PPRE consists of a DR2 site, it was investigated whether statins would also activate PPAR $\alpha$ on a consensus DR1 site. RK13 cells were transfected with a luciferase reporter vector containing six copies of the DR1 site cloned in front of the thymidine kinase promoter in pGL3 (TKpGL3). Statins did not influence reporter activity in the absence of PPAR $\alpha$ cotransfection (Figure 4). As expected, PPAR $\alpha$ cotransfection activated the reporter, and this induction was significantly enhanced by cerivastatin (more than fourfold) and pitavastatin (more than threefold) treatment (Scheffé: $P<0.001$ ) (Figure 4).

Next it was analyzed whether the effect of statins on PPAR $\alpha$ could be reversed by the addition of mevalonate. Mevalonate itself had no effect on PPRE-driven promoter activity both in the absence and presence of cotransfected PPAR $\alpha$. However, pitavastatin- or cerivastatin-induced PPAR $\alpha$ transactivation was abolished in the presence of mevalonate (Figure 4). These data indicate that, similar to apoA-I mRNA, statins exert their action on PPAR $\alpha$ by inhibition of HMG-CoA reductase and that inhibition of the mevalonate pathway leads to induction of PPAR $\alpha$ activity.

Statins activate PPAR $\alpha$ in a promoter-independent manner via its ligand-binding domain. To determine whether statins act via the PPAR $\alpha$ ligand-binding domain (LBD), transfection experiments were performed using chimeric molecules in which the LBD of PPAR $\alpha$ is fused to the DNA-binding domain of the yeast transcription factor Gal4. When RK13 cells were transfected with this construct, treatment with both cerivastatin and pitavastatin resulted in a marked increase of activity of the chimeric Gal4-hPPAR $\alpha$ LBD/luciferase
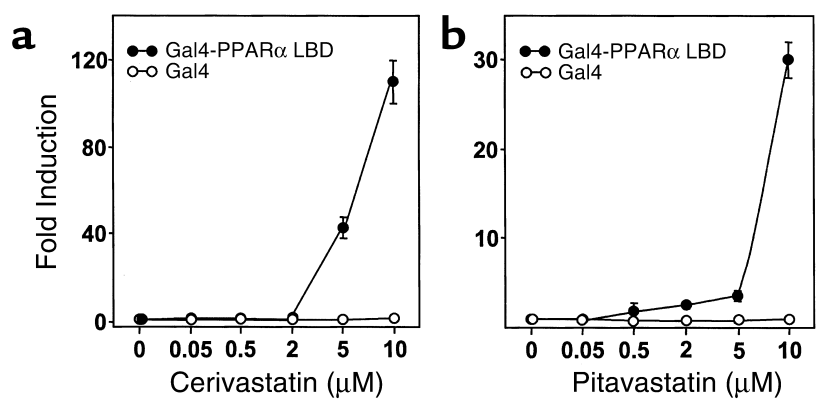

\section{Figure 5}

Statins activate PPAR $\alpha$ by its LBD. RK13 cells were transfected with the pGal5-TK-pGL3 reporter (10 ng) and Gal4-hPPAR $\alpha$ LBD expression plasmids (100 $\mathrm{ng}$ ) with a Renilla luciferase reporter vector as internal control ( $2 \mathrm{ng}$ ). After transfection, cells were further incubated for 40 hours with the indicated concentrations of cerivastatin (a) and pitavastatin (b) or vehicle in DMEM supplemented with $2 \%$ Ultroser SF. Firefly luciferase activities were normalized to Renilla control activities. Values (means $\pm \mathrm{SD}, n=3$ ) are expressed relative to controls. 


\section{Figure 6}

GGpp prevents the activation of PPAR $\alpha$ on PPRE by statins. (a) RK13 cells were transfected with a consensus DR1-driven luciferase reporter vector (10 $\mathrm{ng}$ ) in the presence of pSG5hPPAR $\alpha$ expression vector (30 ng) and a Renilla luciferase reporter vector as internal control $(2 \mathrm{ng})$. Cells were pretreated for 3 hours with ketoprofen (KTP; $50 \mu \mathrm{M}$ ), NDGA $(10 \mu \mathrm{M})$, etomoxir (ETX; $50 \mu \mathrm{M})$, and AACOCF3 (10 $\mu \mathrm{M})$. Medium was removed and replaced by fresh medium supplemented or not with cerivastatin $(5 \mu \mathrm{M})$ for 40 hours. Cerulenin $(5 \mu \mathrm{M})$ and cerivastatin were coincubated for 40 hours. (b) The same experiment as in a was performed with cerivastatin $(5 \mu \mathrm{M})$ and/or CDCA $(10 \mu \mathrm{M})$, squalene (10 $\mu \mathrm{M}), 22(\mathrm{~S})$ and 22(R) HO-cholesterol (HO-Chol.; $10 \mu \mathrm{M}$ ), or vehicle in coincubation for 40 hours after transfection. (c) The same experiment as in a was performed with cerivastatin $(5 \mu \mathrm{M})$ and/or Fpp (G224; 2 and $5 \mu \mathrm{M})$ or farnesol (FOH; $30 \mu \mathrm{M})$, or $\mathrm{FTI}(10 \mu \mathrm{M})$ in coincubation for 40 hours after transfection or vehicle. (d) The same experiment as in a was performed with cerivastatin $(5 \mu \mathrm{M})$ and GGpp (G225; 2 and $5 \mu \mathrm{M})$, or GGTI $(10 \mu \mathrm{M})$, or vehicle in coincubation for 40 hours after transfection. Firefly luciferase activities were normalized to Renilla control activities. Results represent normalized luciferase activity and are expressed as means \pm SD $(n=3)$ relative to the PPAR $\alpha$-only controls (left bar) set as 1 . Values without a common superscript are significantly different, Scheffé: $P<0.001$.
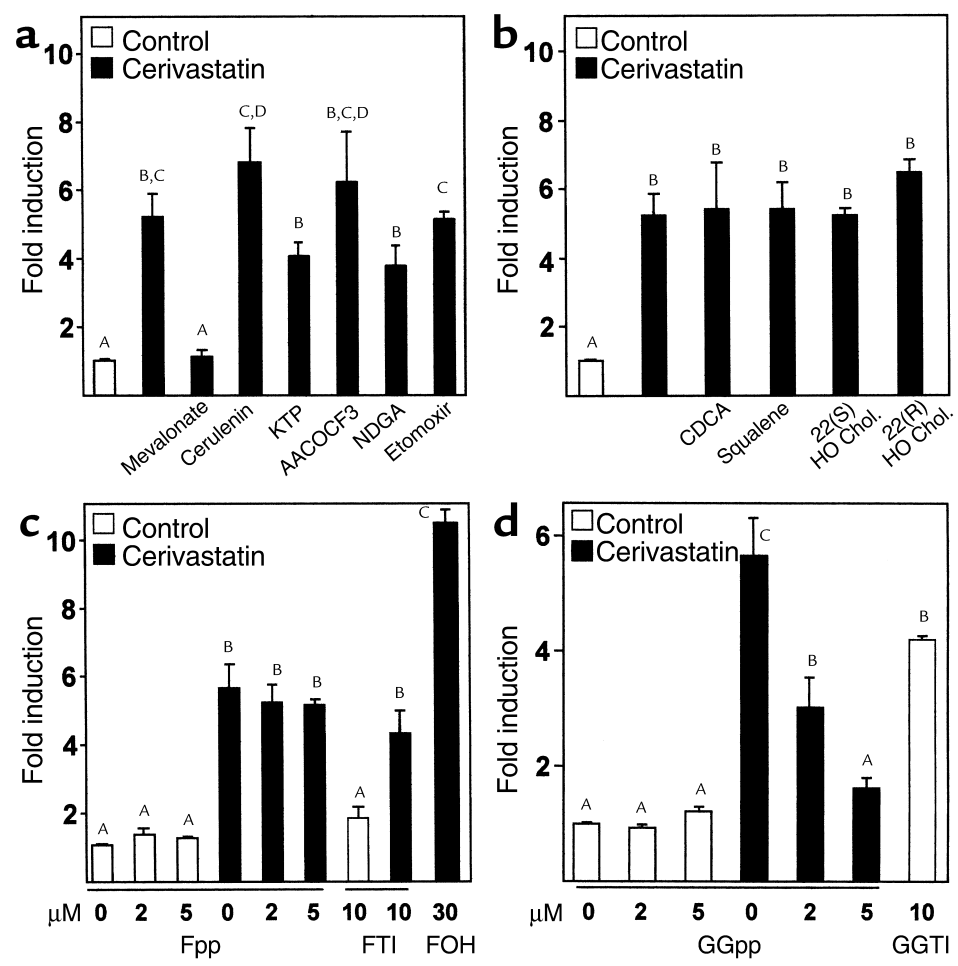

reporter system (Figure 5). By contrast, statins did not influence the activity of a construct containing only the Gal4 DNA-binding domain (DBD) pGal4- $\varnothing$ (Figure 5). These data indicate that statins activate PPAR $\alpha$ by its LBD in a promoter-independent manner.

Statins activate PPAR $\alpha$ by inhibiting the GGPP/Rho signal transduction pathway. Since HMG-CoA reductase inhibition leads to activation of transcription factors of the SREBP family, which induce the expression of lipogenic enzymes (37-39) and as such may generate fatty acid or fatty acid-derived PPAR $\alpha$ ligands (40), the influence of inhibitors of fatty acid synthesis or enzymes known to generate PPAR ligands on statin-induced PPAR $\alpha$ activity was tested. Therefore, the influence of cerulenin, a potent inhibitor of fatty acid synthase (FAS) (41) that may generate long-chain fatty acid PPAR ligands $(23,24$, 42); ketoprofen, an inhibitor of cyclooxygenase that may generate prostaglandin PPAR ligands such as $15-\Delta 12,14$ prostaglandin J2 $(43,44)$; NDGA, an inhibitor of lipoxygenase that may generate PPAR ligands such as 8(S)hydroxyeicosatetraenoic acid (HETE) (45); and AACOCF3, an inhibitor of phospholipase A2 (PLA2) that may generate phospholipid-derived PPAR activators (46) were tested. In addition, the influence of etomoxir, an inhibitor of CPT-1, was also analyzed. Incubation of statin led to a strong activation of the synthetic PPRE-reporter system, which was, however, not significantly affected by pretreatment ( 3 hours) with any of the inhibitors tested (Figure 6a).

Tested next was whether sterol isoprenoids could inhibit statin activation of PPAR $\alpha$ activity. Cholesterolderived metabolites such as bile acids (CDCA) and oxysterols [22(S)- and 22(R)-hydroxycholesterol], or squalene were coincubated to check whether they could repress the statin-mediated induction of PPAR $\alpha$ transactivation. However, none of these compounds prevented the induction of PPAR $\alpha$ activity by statins (Figure $6 b$ ).

Statins also reduce cellular pools of Fpp and GGpp, nonsterol metabolites of mevalonate. The potential involvement of the isoprenoid intermediates Fpp (Figure 6c) and GGpp (Figure 6d) in statin-induced PPAR $\alpha$ activation was evaluated next. Coincubation with GGpp (Figure 6d), but not with Fpp or farnesol (Figure 6c), prevented statin induction of PPAR $\alpha$ activity to an extent similar to that of mevalonate (Figure 6a). Since geranylgeranylation leads to activation of small $\mathrm{G}$ proteins, such as Rho and Rac, the influence of a specific geranylgeranyl transferase I inhibitor (GGTI) on PPAR $\alpha$ activity was tested next. Interestingly, incubation with GGTI resulted in a significant activation of PPAR $\alpha(P<0.001)$ (Figure $6 \mathrm{~d})$. In addition, C3 exoenzyme, which blocks Rho protein activity, enhanced statin induction of PPAR $\alpha$ (twofold; data not shown). As expected, incubation with a farnesyl transferase inhibitor (FTI) did not influence basal nor statininduced PPAR $\alpha$ activity (Figure 6c). These data indicate that statins activate PPAR $\alpha$ by inhibition of geranylgeranylation of small $G$ proteins.

Dominant negative Rho A activates PPAR $\alpha$. Geranylgeranylation of Rho GTP-binding protein family members induces their translocation from the cytosolic compartment to the membrane, leading to their activation. To identify which Rho family member is implicated in the regulation of PPAR $\alpha$, transient cotransfection experiments were performed on a synthetic PPRE in the presence of PPAR $\alpha$ and dominant negative Rho A, 

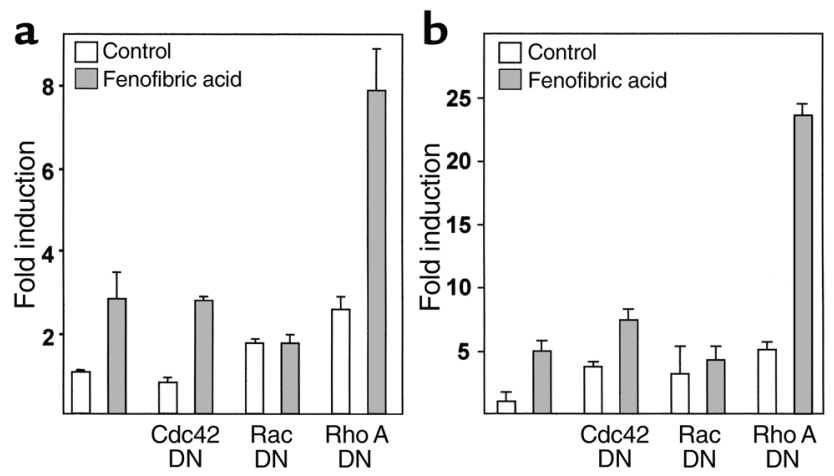

\section{Figure 7}

Dominant negative (DN) Rho A activates PPAR $\alpha$. (a) RK13 cells were transfected with a consensus DR1-driven luciferase reporter vector $(10 \mathrm{ng})$ in the presence of PSG5hPPAR $\alpha$ expression vector $(30 \mathrm{ng})$, a Renilla luciferase reporter vector as internal control (2 ng), and expression vectors of dominant negative Rho A (T19N), Rac (T17N), $\mathrm{Cdc} 42(\mathrm{~T} 17 \mathrm{~N})$, or empty vector (30 ng). After transfection, cells were further incubated for 40 hours with fenofibric acid $(100 \mu \mathrm{M})$ or vehicle in DMEM supplemented with $2 \%$ Ultroser SF. Results represent normalized luciferase activity and are expressed as means \pm SD $(n=3)$ relative to the PPAR $\alpha$ only controls (left bar) set as 1 . (b) RK13 cells were transfected with the pGal5-TK-pGL3 reporter (10 ng) and Gal4-hPPAR $\alpha$ LBD expression plasmids (100 ng), a Renilla luciferase reporter vector as internal control (2 $\mathrm{ng})$, and expression vectors of dominant negative Rho A (T19N), Rac (T17N), Cdc42 $(\mathrm{T} 17 \mathrm{~N})$, or empty vector $(30 \mathrm{ng})$. After transfection, cells were further incubated for 40 hours with fenofibric acid $(100 \mu \mathrm{M})$ or vehicle in DMEM supplemented with $2 \%$ Ultroser SF. Firefly luciferase activities were normalized to Renilla control activities. Values (means \pm SD, $n=3$ ) are expressed relative to controls.

$\mathrm{Cdc} 42$, and Rac1 expression vectors. Whereas cotransfection of dominant negative Cdc42 or Rac did not significantly influence PPAR $\alpha$ activity, dominant negative Rho A induced PPAR $\alpha$, an effect that was enhanced by fibrate treatment $(P<0.001)$ (Figure 7a).

Since statins significantly enhance PPAR $\alpha$ activity via its LBD (Figure 5), we further tested whether inhibition of Rho A also enhances Gal4-PPAR $\alpha$ LBD activity. Whereas cotransfection of dominant negative Cdc42 or Rac did not significantly influence fenofibric acid-induced PPAR $\alpha$ activity, cotransfection with dominant negative Rho A significantly induces this activity $(P<0.001)$ (Figure $7 \mathrm{~b})$.

Cerivastatin treatment decreases PPAR $\alpha$ phosphorylation activity. Since Rho proteins activate protein kinase pathways, it was tested whether statin inhibition of Rho A leads to alterations in PPAR $\alpha$ phosphorylation activity. To test this hypothesis, PPAR $\alpha$ kinase assays were performed on cellular extracts of HepG2 cells treated with either mevalonate or cerivastatin. Most interestingly, cerivastatin treatment led to a marked decrease in PPAR $\alpha$ phosphorylation activity in HepG2 cells (Figure 8a).

Statins and PPAR $\alpha$ agonists activate PPAR $\alpha$ synergistical$l y$. Since PPAR $\alpha$ agonists and statins act by different pathways to activate PPAR $\alpha$, next it was tested whether combination treatment may result in a synergistic induction of PPAR $\alpha$ activation. As expected, in the presence of cotransfected PPAR $\alpha$, cerivastatin, or fenofibric acid alone significantly induced PPAR $\alpha$ activity (Figure 8b). Interestingly, coincubation of cerivastatin and fenofibric acid resulted in a synergistic effect on PPAR $\alpha$ transactivation $(P<0.001)$.

\section{Discussion}

Statins are lipid-lowering drugs widely used in the treatment of hypercholesterolemia. Several intervention trials have demonstrated that statins decrease the incidence of cardiovascular events and improve survival rates both in secondary and primary prevention. In addition to lowering LDL cholesterol, statins reduce triglyceride levels and increase HDL cholesterol. HDL and its major protein constituent apoA-I play a critical role in cholesterol metabolism due to their capacities to eliminate excessive amounts of cholesterol from peripheral arteries and return it back to the liver, the socalled reverse-cholesterol transport pathway. HDL cholesterol levels are inversely correlated with CHD (47), and statins increase HDL cholesterol and apoA-I levels (6). In the present study we studied the molecular mechanism of statin action on apoA-I production.

Statin treatment of human hepatoma cells resulted in a time- and dose-dependent increase of apoA-I mRNA levels. A similar induction of hepatic apoA-I mRNA after statin treatment has been demonstrated previously in vivo in rats (48). In vivo experiments in transgenic apoA-I mice treated with statins revealed no effect on apoA-I mRNA (data not shown), which is likely due to the rapid and almost complete catabolism of

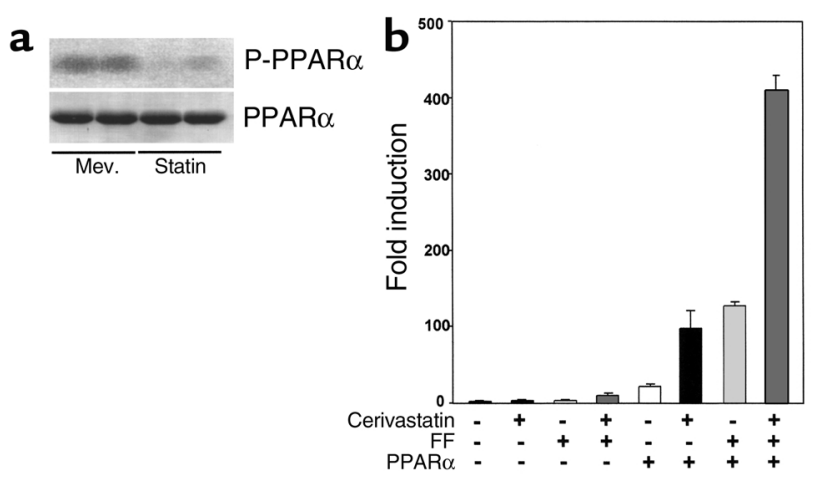

Figure 8

(a) Cerivastatin treatment decreases PPAR $\alpha$ phosphorylation activity. Cellular extracts from HepG2 cells treated with mevalonate ( 3 $\mathrm{mM})$ or cerivastatin $(5 \mu \mathrm{M})$ for 24 hours were incubated with purified PPAR $\alpha$ protein and $\gamma\left[{ }^{32} \mathrm{P}\right]$ ATP and PPAR $\alpha$ phosphorylation determined as described in Methods (top panel). As control for PPAR $\alpha$ loading, the gel was stained with Ponceau S (bottom panel). P-PPAR $\alpha$, phosphorylated PPAR $\alpha$. (b) Statins and PPAR $\alpha$ agonists activate PPAR $\alpha$ synergistically. RK13 cells were transfected with a consensus DR1-driven luciferase reporter vector (10 ng) in the presence of the empty PSG 5 vector or PSG5hPPAR $\alpha$ expression vector (30 ng). Cells were then incubated for 40 hours with cerivastatin ( 5 $\mu \mathrm{M})$ and/or fenofibric acid (FF) $(100 \mu \mathrm{M})$. Firefly luciferase activities were normalized to Renilla control activities. Values (means $\pm \mathrm{SD}$, $n=3$ ) are expressed relative to controls. 


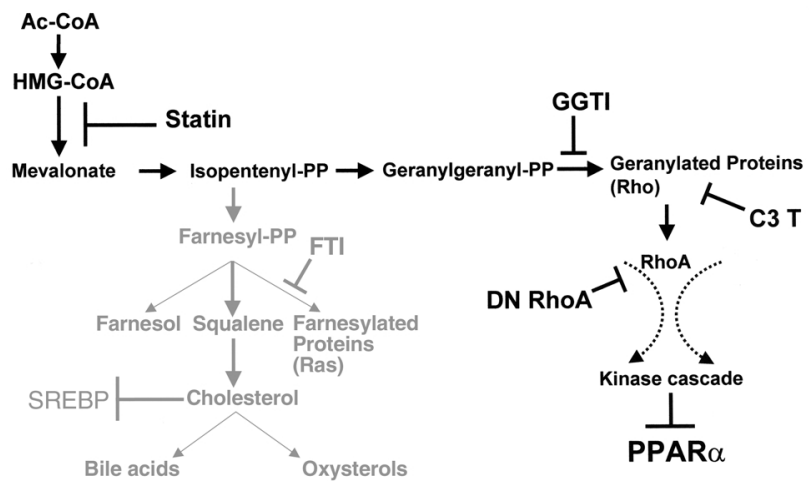

Figure 9

Cross-talk between the statin and PPAR $\alpha$ pathways. C3 T, C 3 transferase; DN, dominant negative. The pathway in black is that implicated in PPAR $\alpha$ regulation by statins. The other pathways of mevalonate metabolism are depicted in gray. PP, pyrophosphate.

cerivastatin in this species (49). The effects of statins on apoA-I expression occur at the transcriptional level since actinomycin D pretreatment blocked the induction. Furthermore, the statin effect depends on downstream products of the mevalonate pathway since mevalonate reversed the increase of apoA-I mRNA levels. To determine the molecular mechanisms involved, transient transfection experiments were performed, and a direct effect on human apoA-I promoter activity was demonstrated. Furthermore, a statin response element was mapped between $-256 \mathrm{bp}$ and $-128 \mathrm{bp}$. Interestingly, a previous study in HepG2 cells demonstrated that cholesterol loading, either by LDL-uptake or addition of free cholesterol, led to an increase in apoAI mRNA levels (50). Since high levels of exogenous cholesterol lead to the downregulation of HMG-CoA reductase, common mechanisms may be the basis of the apoA-I induction by statins and cholesterol loading. This induction of apoA-I gene expression by statins extends the results from previous in vivo studies demonstrating an increase in apoA-I production rate $(6,36)$ after statin administration in humans. Increased expression and production of apoA-I likely contribute, therefore, to the increase of apoA-I and HDL plasma levels observed after statin therapy.

A statin response element was mapped to the A site, which also contains a functional PPRE (31). The induction of human apoA-I gene expression by fibrates, another class of hypolipidemic drugs, occurs by activation of PPAR $\alpha$, which binds to this element. Interestingly, fibrates and statins share a number of pharmacological properties. Both classes of drugs reduce triglyceride and increase HDL levels and exert antithrombotic and anti-inflammatory actions in the vascular wall $(25,51)$. These observations led us to test whether statins could activate PPAR $\alpha$. Results from cotransfection experiments with PPAR $\alpha$ in HepG2 cells demonstrate that statins act directly on the apoAI A site via PPAR $\alpha$. In addition, in RK13 cells that are devoid of PPAR $\alpha$, statins activate a synthetic PPRE- driven promoter only in the presence of cotransfected PPAR $\alpha$. These results suggest a role for this transcription factor in the statin regulation of apoA-I gene expression and clearly establish a cross-talk between the PPAR $\alpha$ and statin-signaling pathways. Since mevalonate addition reverses the PPAR $\alpha$ activation by statins, it can be concluded that statins are not direct ligands, but rather that downstream products of the mevalonate pathway inhibit PPAR $\alpha$ activity. Transfection experiments using the LBD of PPAR $\alpha$ fused to the yeast transcription factor Gal4 DBD indicate that statins induce the transactivation capacity of PPAR $\alpha$ in a general, promoter-independent manner and suggest that statin treatment may result in the generation of PPAR $\alpha$ ligands or may increase the activity of the DBD of PPAR $\alpha$.

Mevalonate is a key intermediate in the de novo synthesis of both sterol and nonsterol isoprenoids. The majority of mevalonate is converted to cholesterol, which is a precursor of steroid hormones, bile acids, vitamin D, and a wide variety of oxysterols. Furthermore, a variety of nonsteroidal isoprenoid products are formed from mevalonate. Cholesterol depletion induced by statins triggers the cleavage of the cholesterol-sensitive transcription factors, called SREBPs. Studies in adipocytes revealed that ADD1/SREBP-1, a transcription factor participating in adipose tissue differentiation, leads to the production of endogenous ligands for PPAR $\gamma$ (40). These intermediates are lipid molecules that bind directly to PPAR $\gamma$, since they displaced the binding of synthetic ligands such as thiazolidinediones. Interestingly, SREBP activation leads to the induction of lipogenic enzymes such as FAS and thus may lead to the production of fatty acids, which are ligands of PPAR $\alpha(23,24,42)$. In addition, PPAR ligands may be produced by oxidation of endogenous fatty acids by lipoxygenases $(24,42,45,52)$, or cyclooxygenases $(43,44)$, or by degradation of phospholipids by phospholipase A2, which liberates fatty acids (46). This raised the question of whether PPAR $\alpha$ ligands might be generated through activation of these pathways. However, experiments to reverse statin action on PPAR $\alpha$ activity using potent inhibitors of these enzymes were without effect on statin-induced PPAR $\alpha$ activity. Furthermore, compounds derived from the cholesterol biosynthetic pathway, including sterols, squalene, or cholesterol metabolites such as bile acids or oxysterols, did not modulate statin-induced PPAR $\alpha$ activity. Thus these data provide evidence that statins activate PPAR $\alpha$ via a pathway other than the sterol or SREBP pathways. Mevalonate is not only a precursor for cholesterol synthesis but also is a precursor of nonsteroidal isoprenoid compounds. Fpp and GGpp are substrates for the posttranslational prenylation of proteins (53-55). Farnesol is a Fpp-derived metabolite that has been shown recently to induce PPAR $\alpha$ activity and thereby influence keratinocyte differentiation (56). However, our results demonstrate that statin action on PPAR $\alpha$ was prevented by GGpp, but not Fpp nor farnesol, sug- 
gesting that geranylgeranyl-modified intermediates may antagonize PPAR $\alpha$. Fpp and GGpp are implicated in membrane translocation, leading to the activation of a variety of proteins, including Ras and Rho GTPbinding proteins, respectively $(57,58)$. Rho A, Rho B, $\mathrm{Rac}$, and $\mathrm{Cdc} 42$ are the major substrates for posttranslational modification by geranylgeranylation, which leads to their activation and membrane translocation. This process has been shown to be inhibited by statins in SMCs indicating a direct effect of statins on the vascular wall via inhibition of Rho geranylgeranylation (59). After posttranslational modification by geranylgeranylation (18), the Rho family of small GTP-binding proteins can be inactivated by treatment with $\mathrm{C} 3$ exoenzyme, which selectively ADP-ribosylates lowmolecular-weight $\mathrm{G}$ proteins of the Rho $\mathrm{A}$ and $\mathrm{B}$ subfamily, rendering them biologically inactive (60). C3 exoenzyme treatment enhanced statin-induction of PPAR $\alpha$ transactivation (data not shown), further pointing to the implication of Rho proteins in the statin activation of PPAR $\alpha$. Since Rho A, but not Cdc 42 or Rac dominant negative proteins, enhances PPAR $\alpha$ activity and, more specifically, the PPAR $\alpha$ LBD, it is likely that the effects of statins on PPAR $\alpha$ are mediated by Rho A. Downstream targets of Rho family proteins have just begun to be identified, and the molecular mechanisms by which Rho proteins may regulate gene expression are not clearly understood. Posttranslationally modified Rho proteins control cytoskeletal reorganization, motility, and cell growth (61). Rho, Rac, and $\mathrm{Cdc} 42$ have been reported to regulate the c-Jun $\mathrm{NH}_{2}$ terminal kinase (JNK), and the p38 MAP kinase (MAPK) cascades (62-64). PPAR $\alpha$ activity is modulated by phosphorylation, resulting in either enhanced or lowered transcriptional activity (65). In this study, we show that statins decrease the phosphorylation of PPAR $\alpha$. Interestingly, a recent study demonstrated downregulation of PPAR $\alpha$ activity after activation of the MAPK pathway (66). However, MAPK sites in PPAR $\alpha$ were mapped in the $\mathrm{NH}_{2}$-terminal part of the protein (67). Since statins induce PPAR $\alpha$ LBD by its activity, they likely act through a novel mechanism. Further studies are required to delineate the molecular mechanism of PPAR $\alpha$ regulation by Rho A. The GGPP pathway has already been implicated in mediating the antithrombotic and anti-inflammatory effects of statins in SMCs and macrophages $(16,21)$. Our data provide evidence that the GGPP pathway is also implicated in the effects of statins acting on PPAR $\alpha$. Since PPAR $\alpha$ exerts potent anti-inflammatory activities in vascular cells $(27,68)$, we speculate that the reported anti-inflammatory activities of statins are, at least in part, mediated by PPAR $\alpha$ activation (Figure 9).

Previous studies have demonstrated induction of PPAR $\gamma$ activity by statins through the generation of ligands after SREBP activation (40). Furthermore, PPAR $\gamma$ transcription has been shown to be induced by statins by a SREBP response element in the PPAR $\gamma$ promoter (69). However, this is the first time that a cross-talk of the PPAR $\alpha$ and statin-signaling pathways is shown. Furthermore, we show that PPAR $\alpha$ activation by statins occurs through a completely different molecular mechanism, implicating the GGPP pathway and prenylation of Rho family proteins. Moreover, we demonstrate that simultaneous treatment with statins and fibrate PPAR $\alpha$ ligands results in a synergistic effect on PPAR $\alpha$ transactivation. Thus PPAR $\alpha$ is an important molecular target for the two major classes of hypolipidemic drugs. Together, these data provide a molecular rationale for combination therapy with statins and fibrates in the treatment of CHD.

\section{Acknowledgments}

The authors thank Dean W. Hum for helpful comments on this manuscript. This work was supported by grants from INSERM, Universite de Lille II, Institut Pasteur de Lille, and Kowa Negma. G. Martin was supported by a grant from Bayer A.G.

1. Gotto, A.M., Jr., and Grundy, S.M. 1999. Lowering LDL cholesterol: questions from recent meta-analyses and subset analyses of clinical trial data issues from the Interdisciplinary Council on Reducing the Risk for Coronary Heart Disease, ninth Council meeting. Circulation. 99:E1-E7.

2. Hebert, P.R., Gaziano, J.M., Chan, K.S., and Hennekens, C.H. 1997. Cholesterol lowering with statin drugs, risk of stroke, and total mortality. An overview of randomized trials. JAMA. 278:313-321.

3. Brown, M.S., and Goldstein, J.L. 1997. The SREBP pathway: regulation of cholesterol metabolism by proteolysis of a membrane-bound transcription factor. Cell. 89:331-340.

4. Superko, H.R. 1996. Beyond LDL cholesterol reduction. Circulation. 94:2351-2354.

5. Vega, G.L., and Grundy, S.M. 1998. Effect of statins on metabolism of apoB-containing lipoproteins in hypertriglyceridemic men. Am. J. Cardiol. 81:36B-42B.

6. Schaefer, J.R., et al. 1999. Metabolic basis of high density lipoproteins and apolipoprotein A-I increase by HMG-CoA reductase inhibition in healthy subjects and a patient with coronary artery disease. Atherosclerosis. 144:177-184.

7. Stein, O., and Stein, Y. 1999. Atheroprotective mechanisms of HDL. Atherosclerosis. 144:285-301.

8. Miller, G.J., and Miller, N.E. 1975. Plasma-high-density-lipoprotein concentration and development of ischaemic heart-disease. Lancet. 1:16-19.

9. Gordon, T., Castelli, W.P., Hjortland, M.C., Kannel, W.B., and Dawber, T.R. 1977. High density lipoprotein as a protective factor against coronary heart disease. The Framingham Study. Am. J. Med. 62:707-714.

10. Vega, G.L., and Grundy, S.M. 1996. Hypoalphalipoproteinemia (low high density lipoprotein) as a risk factor for coronary heart disease. Curr. Opin. Lipidol. 7:209-216.

11. Rubin, E.M., Ishida, B.Y., Clift, S.M., and Krauss, R.M. 1991. Expression of human apolipoprotein A-I in transgenic mice results in reduced plasma levels of murine apolipoprotein A-I and the appearance of two new high density lipoprotein size subclasses. Proc. Natl. Acad. Sci. USA. 88:434-438.

12. Duverger, N., et al. 1996. Inhibition of atherosclerosis development in cholesterol-fed human apolipoprotein A-I-transgenic rabbits. Circulation. 94:713-717.

13. Bellosta, S., et al. 2000. Pleiotropic effects of statins in atherosclerosis and diabetes. Diabetes Care. 23(Suppl.):B72-B78.

14. Davignon, J., and Laaksonen, R. 1999. Low-density lipoprotein-independent effects of statins. Curr. Opin. Lipidol. 10:543-559.

15. Bellosta, S., et al. 1998. Direct vascular effects of HMG-CoA reductase inhibitors. Atherosclerosis. 137(Suppl.):S101-S109.

16. Bellosta, S., et al. 1998. HMG-CoA reductase inhibitors reduce MMP-9 secretion by macrophages. Arterioscler. Thromb. Vasc. Biol. 18:1671-1678.

17. Takeuchi, S., et al. 2000. Cerivastatin suppresses lipopolysaccharideinduced ICAM-1 expression through inhibition of Rho GTPase in BAEC. Biochem. Biophys. Res. Commun. 269:97-102.

18. Essig, M., et al. 1998. 3-Hydroxy-3-methylglutaryl coenzyme A reductase inhibitors increase fibrinolytic activity in rat aortic endothelial cells. Role of geranylgeranylation and Rho proteins. Circ. Res. 83:683-690.

19. Colli, S., et al. 1997. Vastatins inhibit tissue factor in cultured human macrophages. A novel mechanism of protection against atherothrombosis. Arterioscler. Thromb. Vasc. Biol. 17:265-272.

20. Maron, D.J., Fazio, S., and Linton, M.F. 2000. Current perspectives on 
statins. Circulation. 101:207-213

21. Bourcier, T., and Libby, P. 2000. HMG CoA reductase inhibitors reduce plasminogen activator inhibitor-1 expression by human vascular smooth muscle and endothelial cells. Arterioscler. Thromb. Vasc. Biol. 20:556-562.

22. Issemann, I., and Green, S. 1990. Activation of a member of the steroid hormone receptor superfamily by peroxisome proliferators. Nature. 347:645-650.

23. Forman, B.M., Chen, J., and Evans, R.M. 1997. Hypolipidemic drugs, polyunsaturated fatty acids, and eicosanoids are ligands for peroxisome proliferator-activated receptors alpha and delta. Proc. Natl. Acad. Sci. USA. 94:4312-4317.

24. Kliewer, S.A., et al. 1997. Fatty acids and eicosanoids regulate gene expression through direct interactions with peroxisome proliferator-activated receptors alpha and gamma. Proc. Natl. Acad. Sci. USA. 94:4318-4323.

25. Pineda Torra, I., Gervois, P., and Staels, B. 1999. Peroxisome proliferatoractivated receptor alpha in metabolic disease, inflammation, atherosclerosis and aging. Curr. Opin. Lipidol. 10:151-159.

26. Staels, B., et al. 1998. Mechanism of action of fibrates on lipid and lipoprotein metabolism. Circulation. 98:2088-2093.

27. Staels, B., et al. 1998. Activation of human aortic smooth-muscle cells is inhibited by PPARalpha but not by PPARgamma activators. Nature. 393:790-793

28. Delerive, P., et al. 1999. Peroxisome proliferator-activated receptor activators inhibit thrombin-induced endothelin-1 production in human vascular endothelial cells by inhibiting the activator protein-1 signaling pathway. Circ. Res. 85:394-402.

29. Staels, B., van Tol, A., Andreu, T., and Auwerx, J. 1992. Fibrates influence the expression of genes involved in lipoprotein metabolism in a tissueselective manner in the rat. Arterioscler. Thromb. 12:286-294.

30. Berthou, L., et al. 1996. Opposite regulation of human versus mouse apolipoprotein A-I by fibrates in human apolipoprotein A-I transgenic mice. J. Clin. Invest. 97:2408-2416.

31. Vu-Dac, N., et al. 1994. Negative regulation of the human apolipoprotein A-I promoter by fibrates can be attenuated by the interaction of the peroxisome proliferator-activated receptor with its response element. J. Biol. Chem. 269:31012-31018.

32. Gervois, P., et al. 1999. A truncated human peroxisome proliferator-activated receptor alpha splice variant with dominant negative activity. Mol. Endocrinol. 13:1535-1549.

33. Raspe, E., et al. 1999. Modulation of rat liver apolipoprotein gene expression and serum lipid levels by tetradecylthioacetic acid (TTA) via PPARalpha activation. J. Lipid Res. 40:2099-2110.

34. Gauthier-Rouvière, C., et al. 1998. RhoG GTPase controls a pathway that independently activates Rac1 and Cdc42Hs. Mol. Biol. Cell. 9:1379-1394.

35. Chinetti, G., et al. 1998. Activation of proliferator-activated receptors $\alpha$ and $\gamma$ induces apoptosis of human monocyte-derived macrophages. J. Biol. Chem. 273:25573-25580.

36. Ginsberg, H.N., Ngai, C., and Ranmakrishnan, R. 1991. Lovastatin increases apolipoprotein A-I levels in subjects with isolated reductions in high density lipoproteins. Circulation. 84:II-140. (Abstr.)

37. Kim, J.B., and Spiegelman, B.M. 1996. ADD1/SREBP1 promotes adipocyte differentiation and gene expression linked to fatty acid metabolism. Genes Dev. 10:1096-1107.

38. Shimano, H., et al. 1996. Overproduction of cholesterol and fatty acids causes massive liver enlargement in transgenic mice expressing truncated SREBP-1a. J. Clin. Invest. 98:1575-1584.

39. Shimano, H., et al. 1999. Sterol regulatory element-binding protein-1 as a key transcription factor for nutritional induction of lipogenic enzyme genes. J. Biol. Chem. 274:35832-35839.

40. Kim, J.B., Wright, H.M., Wright, M., and Spiegelman, B.M. 1998. ADD1/SREBP1 activates PPARgamma through the production of endogenous ligand. Proc. Natl. Acad. Sci. USA. 95:4333-4337.

41. Omura, S. 1976. The antibiotic cerulenin, a novel tool for biochemistry as an inhibitor of fatty acid synthesis. Bacteriol. Rev. 40:681-697.

42. Yu, K., et al. 1995. Differential activation of peroxisome proliferator-activated receptors by eicosanoids. J. Biol. Chem. 270:23975-23983.

43. Kliewer, S.A., et al. 1995. A prostaglandin J2 metabolite binds peroxisome proliferator-activated receptor gamma and promotes adipocyte differentiation. Cell. 83:813-819.

44. Forman, B.M., et al. 1995. 15-Deoxy-delta 12, 14-prostaglandin J2 is a ligand for the adipocyte determination factor PPAR gamma. Cell. 83:803-812.
45. Huang, J.T., et al. 1999. Interleukin-4-dependent production of PPARgamma ligands in macrophages by 12/15-lipoxygenase. Nature. 400:378-382.

46. Delerive, P., et al. 2000. Oxidized phospholipids activate PPARalpha in a phospholipase A2-dependent manner. FEBS Lett. 471:34-38.

47. Schaefer, E.J. 1984. Clinical, biochemical, and genetic features in familial disorders of high density lipoprotein deficiency. Arteriosclerosis. 4:303-322.

48. Mitchell, A., Fidge, N., and Griffiths, P. 1993. The effect of the HMG-CoA reductase inhibitor simvastatin and of cholestyramine on hepatic apolipoprotein mRNA levels in the rat. Biochim. Biophys. Acta. 1167:9-14

49. Boberg, M., et al. 1998. Biotransformation of cerivastatin in mice, rats, and dogs in vivo. Drug. Metab. Dispos. 26:640-652

50. Monge, J.C., Hoeg, J.M., Law, S.W., and Brewer, H.B., Jr. 1989. Effect of low density lipoproteins, high density lipoproteins, and cholesterol on apolipoprotein A-I mRNA in Hep G2 cells. FEBS Lett. 243:213-217.

51. Rosenson, R.S., and Tangney, C.C. 1998. Antiatherothrombotic properties of statins: implications for cardiovascular event reduction. JAMA. 279:1643-1650.

52. Krey, G., et al. 1997. Fatty acids, eicosanoids, and hypolipidemic agents identified as ligands of peroxisome proliferator-activated receptors by coactivator-dependent receptor ligand assay. Mol. Endocrinol. 11:779-791.

53. Flint, O.P., Masters, B.A., Gregg, R.E., and Durham, S.K. 1997. HMG CoA reductase inhibitor-induced myotoxicity: pravastatin and lovastatin inhibit the geranylgeranylation of low-molecular-weight proteins in neonatal rat muscle cell culture. Toxicol. Appl. Pharmacol. 145:99-110.

54. Danesi, R., McLellan, C.A., and Myers, C.E. 1995. Specific labeling of isoprenylated proteins: application to study inhibitors of the post-translational farnesylation and geranylgeranylation. Biochem. Biophys. Res. Com mun. 206:637-643

55. Sinensky, M., and Lutz, R.J. 1992. The prenylation of proteins. Bioessays. 14:25-31.

56. Hanley, K., et al. 2000. Farnesol stimulates differentiation in epidermal keratinocytes via PPARalpha. J. Biol. Chem. 275:11484-11491.

57. Goldstein, J.L., and Brown, M.S. 1990. Regulation of the mevalonate pathway. Nature. 343:425-430.

58. Casey, P.J. 1995. Protein lipidation in cell signaling. Science. 268:221-225.

59. Laufs, U., Marra, D., Node, K., and Liao, J.K. 1999. 3-Hydroxy-3-methylglutaryl-CoA reductase inhibitors attenuate vascular smooth muscle proliferation by preventing rho GTPase-induced down-regulation of p27(Kip1). J. Biol. Chem. 274:21926-21931.

60. Aktories, K. 1997. Bacterial toxins that target Rho proteins. J. Clin. Invest. 99:827-829.

61. Hall, A. 1998. Rho GTPases and the actin cytoskeleton. Science 279:509-514

62. Coso, O.A., et al. 1995. The small GTP-binding proteins Rac1 and Cdc42 regulate the activity of the JNK/SAPK signaling pathway. Cell. 81:1137-1146.

63. Minden, A., Lin, A., Claret, F.X., Abo, A., and Karin, M. 1995. Selective activation of the JNK signaling cascade and c-Jun transcriptional activity by the small GTPases Rac and Cdc42Hs. Cell. 81:1147-1157.

64. Teramoto, H., et al. 1996. The small GTP-binding protein rho activates cJun $\mathrm{N}$-terminal kinases/stress-activated protein kinases in human kidney 293 T cells. Evidence for a Pak-independent signaling pathway. J. Biol. Chem 271:25731-25734.

65. Shalev, A., et al. 1996. The peroxisome proliferator-activated receptor alpha is a phosphoprotein: regulation by insulin. Endocrinology. 137:4499-4502.

66. Barger, P.M., Brandt, J.M., Leone, T.C., Weinheimer, C.J., and Kelly, D.P. 2000. Deactivation of peroxisome proliferator-activated receptor-alpha during cardiac hypertrophic growth. J. Clin. Invest. 105:1723-1730.

67 Juge-Aubry, C.E., et al. 1999. Regulation of the transcriptional activity of the peroxisome proliferator-activated receptor alpha by phosphorylation of a ligand-independent trans-activating domain. J. Biol. Chem. 274:10505-10510

68. Zhou, Y.C., and Waxman, D.J. 1999. Cross-talk between janus kinase-signal transducer and activator of transcription (JAK-STAT) and peroxisome proliferator-activated receptor-alpha (PPARalpha) signaling pathways. Growth hormone inhibition of PPAR alpha transcriptional activity mediated by stat5b. J. Biol. Chem. 274:2672-2681.

69. Fajas, L., et al. 1999. Regulation of peroxisome proliferator-activated receptor gamma expression by adipocyte differentiation and determination factor 1 /sterol regulatory element binding protein 1 : implications for adipocyte differentiation and metabolism. Mol. Cell. Biol. 19:5495-5503. 